\title{
Individual differences in trait aggression modulate neural sensitivity to unpleasant stimuli: evidence from an ERP study.
}

\author{
Xiaojun $\mathrm{Li}^{1}$, Zongkui Zhou ${ }^{2}$, Shanping $\mathrm{He}^{3}$, Zhenhong Wang ${ }^{4 *}$ \\ ${ }^{1}$ Department of Education, the Research Base of Hanshui Culture, Hanjiang Normal University, PR China \\ ${ }^{2}$ Key Laboratory of Adolescent Psychology and Behavior of the Network (CCNU) Ministry of Education, Central China \\ Normal University, PR China \\ ${ }^{3}$ Faculty of Pre-school Education, Shaanxi Xueqian Normal University, PR China \\ ${ }^{4}$ School of Psychology, Shaanxi Normal University, PR China
}

\begin{abstract}
Using ERP measures, the present study investigated the neural mechanism of the interaction between trait aggression and neural sensitivity to unpleasant stimuli. Event-related potentials (ERPs) were recorded for pleasant and unpleasant stimuli when subjects were required to categorize the emotion of stimuli. The ERP result showed a significant interaction between aggression and emotion during 450-600 ms interval. Unpleasant stimuli elicited more pronounced positive deflection than pleasant stimuli in low aggressive subjects whereas this was not observed in high aggressive subjects. This suggests that trait aggression influences the neural sensitivity to unpleasant stimuli.
\end{abstract}

Keywords: Trait aggression, Neural sensitivity, Unpleasant stimuli, Event-related potential (ERP)

Accepted on April 19, 2017

\section{Introduction}

Considerable studies suggest that individual differences in neural sensitivity to unpleasant stimuli exist and that a major proportion of the variance can be explained in personality [1-3]. Thus, understanding the neural mechanism of the interaction between personality and neural sensitivity to unpleasant stimuli is important to expand our knowledge of individual differences in social behaviour. The present study aimed to examine the effects of individual differences in trait aggression on the neural sensitivity to unpleasant stimuli.

The study investigated whether individual differences in trait aggression modulate the neural sensitivity to unpleasant stimuli compared with pleasant stimuli [4-5]. Unpleasant stimuli elicited increased positive deflection than pleasant stimuli during 450-600 ms interval. More importantly, a significant interaction between aggression and emotion was observed during 450-600 ms interval. Unpleasant stimuli elicited more pronounced positive deflection than pleasant stimuli in low aggressive subjects whereas this was not observed in high aggressive subjects.

The neural sensitivity to unpleasant stimuli is affected by trait aggression. Specifically, in the present study unpleasant stimuli in low trait aggressive individuals would probably evoke enhanced ERP reactions than did pleasant stimuli at several temporal points. In contrast, the ERP effects of emotion would be smaller, or even absent in high trait aggressive subjects.
This may be manifested by the similar ERPs elicited by unpleasant and pleasant stimuli in high trait aggressive individual.

\section{Methods}

\section{Subjects}

A total of 300 undergraduate students completed Buss-Perry questionnaire. Participants whose trait aggression scores were above 90 (75 percentile) were grouped as high aggressive individuals, whereas those of the subjects of low aggressive group were equal or less than 60 (25 percentile). Finally, 28 volunteers were included, 14 in the high aggressive group (six male and eight female), and 14 in the low aggressive group (seven male and seven female). The average age for participants in the high aggressive group was 21 years $(\mathrm{SD}=2.1)$ and that for participants in the low aggressive group was 20 years $(\mathrm{SD}=1.9)$. All subjects were with right-handed and they had normal or corrected to normal visions.

\section{Stimuli}

The words used in the experiment were taken from a collection of 526 words. They were validated by another group of subjects $(\mathrm{N}=30)$. The subjects rated the degree of emotional experience by a 5 point scale (from $1=$ excitement to $5=$ calm) for the collection of words. Basing on the subjects' assessment 
and their emotion arousal signaled by the words, 50 pleasant and 50 unpleasant words were chosen for the experiment.

\section{ERP recording and analysis}

The EEG was recorded from 64 scalp sites using tin electrodes mounted in an elastic cap (Brain Products), with the references on the left and right mastoids and a ground electrode on the medial frontal aspect. The vertical electrooculograms (EOGs) were recorded supra- and infra-orbitally at the left eye. The horizontal EOG was recorded from the left versus right orbital rim. The EEG and EOG were amplified using a 0.01 16 Hz bandpass and continuously sampled at $500 \mathrm{~Hz} /$ channel. All inter-electrode impedance was maintained below $10 \mathrm{k} \Omega$. Averaging of ERPs was computed off-line; Trials with EOG artifacts (mean EOG voltage exceeding $\pm 80 \mu \mathrm{V}$ ) and those contaminated with artifacts due to amplifier clipping, peak-topeak deflection exceeding $\pm 80 \mu \mathrm{V}$ were excluded from averaging.

EEG activity for correct responses during each stimulus condition was overlapped and averaged separately. ERP waveforms were time-locked to the onset of stimuli and the average epoch was $800 \mathrm{~ms}$, including a $200 \mathrm{~ms}$ pre-stimulus baseline. As shown by the averaged ERP waveforms, the amplitude differences between unpleasant and pleasant conditions started at about $200 \mathrm{~ms}$. Thus, we selected the following 7 electrode sites for statistical analysis: $\mathrm{Fz}, \mathrm{FCz}, \mathrm{Cz}$, $\mathrm{CPz}, \mathrm{Pz}, \mathrm{POz}$, and 0z. Averaged ERP amplitudes were measured and analyzed within LPC (450-600ms) intervals. A three-way repeated measure Analysis of Variance (ANOVA) on the amplitude of each component was conducted with emotion condition(two levels: unpleasant, pleasant), and electrode sites as within-subjects factors, and trait aggression as betweensubjects factor. The degrees of freedom of the F-ratio were corrected according to the Greenhouse-Geisser method in all these analysis (Figure 1).

\section{Results}

\section{Behavioral results}

A two-way ANOVA on accuracy data (emotion as withinsubjects factor whereas aggression as between-subjects factor) demonstrated a significant emotion effect $[F(1,26)=14.389$, $\mathrm{P}<0.01]$. The accuracy was lower during unpleasant condition $(91.3 \%)$ than that during pleasant condition (96.3\%). A twoway ANOVA on RT data (emotion as within-subjects factor whereas aggression as between-subjects factor) demonstrated a significant emotion effect $[\mathrm{F}(1,26)=6.663, \mathrm{P}<0.05]$. The reaction time was longer during unpleasant condition (694.48 $\mathrm{ms}$ ) than that during pleasant condition $(673.329 \mathrm{~ms})$.

LPC (450-600 ms): A repeated measures ANOVA conducted on the amplitudes demonstrated significant main effect of emotion $[\mathrm{F}(1,26)=5.053, \mathrm{P}<0.05]$ and electrode site $[\mathrm{F}$ $(6,156)=10.414 \mathrm{P}<0.001]$. The largest positive deflection was at Fz. the amplitudes were larger during pleasant conditions than
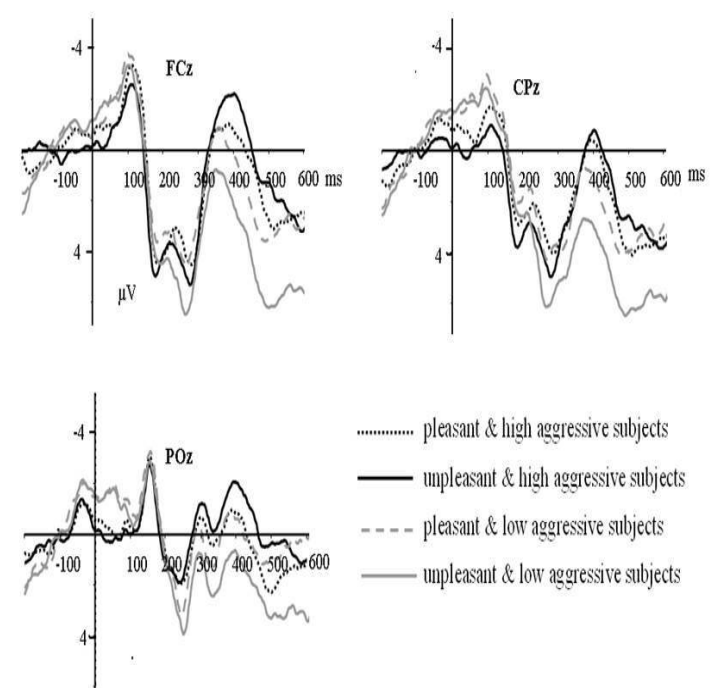

Figure 1. The averaged event-related potentials by unpleasant stimuli at $\mathrm{FCz}, \mathrm{CPz}$ and $\mathrm{POz} \mathrm{ERP}$ analysis.

during unpleasant conditions. In addition, a significant interaction between emotion and aggression $[F(1,26)=6.322$, $\mathrm{P}<0.05]$ was found. Simple effects analyses of the interaction effect between aggression and valence demonstrated a significant emotion effect in low aggressive subjects [F $(1,13)$ $=16.413, \mathrm{P}<0.001]$, and unpleasant condition elicited smaller amplitudes than pleasant condition. However, no significant emotion effect was found in high aggressive subjects $[\mathrm{F}(1,13)$ $=0.752, \mathrm{P}=0.872]$.

\section{Conclusion}

The present study observed significant modulating effects of aggression on neural sensitivity to unpleasant stimuli. This promotes our understanding of the interaction between aggression and neural sensitivity to unpleasant stimuli, suggesting that high and low aggressive individuals have different neural sensitivities to unpleasant stimuli.

\section{Acknowledgements}

This work was financially supported by the Research Base of Hanshui Culture (2015A06).

\section{References}

1. Barth Olow BD, Bushman BJ, Sestir MA.Chronic violent video game exposure and desensitization to violence: behavioral and event-related brain potential data. J Exp Soc Psychol 2006; 42: 532-539.

2. Beaver JD, Lawrence AD, Passamonti L, Calder AJ. Appetitive motivation predicts the neural response to viewing facial signals of aggression. J of Neurosci 2008; 28: 2719- 2725 .

3. Bediou B, Eimer M, D'Amato T, Hauk O, Calder AJ. In the eye of the beholder: Individual differences in reward-drive 
Individual differences in trait aggression modulate neural sensitivity to unpleasant stimuli: evidence from an ERP study.

modulate early frontocentral ERPs to angry faces. "Correspondence to Neuropsychol 2009; 47: 825-834.

4. Dietrich DE, Emrich HE, Waller C, Wiering BM. Emotion/ cognition-coupling in word recognition memory of depressive patients: an event-related potential study. Psych Res 2000; 96: 15-29.

5. Matheson E, Jahoda A. Emotional understanding in aggressive and nonaggressive individuals with mild or moderate mental retardation. Am J Ment Retard 2005; 110: 57-67.

Zhenhong Wang

School of Psychology,

Shaanxi Normal University,

PR China 